\title{
Extracting the Cosmological Constant from the Wheeler DeWitt Equation in a Modified Gravity Theory
}

\author{
Remo Garattini
}

\begin{abstract}
We discuss how to extract information about the cosmological constant from the Wheeler-DeWitt equation, considered as an eigenvalue of a Sturm-Liouville problem. A generalization to a $f(R)$ theory is taken under examination. The equation is approximated to one loop with the help of a variational approach with Gaussian trial wave functionals. We use a zeta function regularization to handle with divergences. A renormalization procedure is introduced to remove the infinities together with a renormalization group equation.
\end{abstract}

Università degli Studi di Bergamo, Facoltà di Ingegneria, Viale Marconi 5, 24044 Dalmine (Bergamo) ITALY.

INFN - sezione di Milano, Via Celoria 16, Milan, Italy

E-mail: remo.garattini@unibg.it

\section{Introduction}

The Einstein's field equations represent a fundamental set of information regarding the laws of space-time. They are represented by

$$
R_{\mu \nu}-\frac{1}{2} g_{\mu \nu} R+\Lambda_{c} g_{\mu \nu}=\kappa T_{\mu \nu}
$$

where $T_{\mu \nu}$ is the energy-momentum tensor of some matter fields, $\kappa=8 \pi G$ with $G$ the Newton's constant and $\Lambda_{c}$ is the cosmological constant . The sourceless version of Eqs.(1) is simply

$$
R_{\mu \nu}-\frac{1}{2} g_{\mu \nu} R+\Lambda_{c} g_{\mu \nu}=0
$$

It is well known that there exists a huge discrepancy between the observed[1] and the computed value of the cosmological constant. It amounts approximately to a factor of 120 orders of magnitude: this is the cosmological constant problem. One possible approach to such a problem is given by the Wheeler-DeWitt equation (WDW) 2]. The WDW equation can be extracted from the Einstein's field equations with and without matter fields in a very simple way. If we introduce a time-like unit vector $u^{\mu}$ such that $u \cdot u=-1$, then after a little rearrangement, we get $t$

$$
\mathcal{H}=(2 \kappa) G_{i j k l} \pi^{i j} \pi^{k l}-\frac{\sqrt{g}}{2 \kappa}\left({ }^{3} R-2 \Lambda_{c}\right)=0 .
$$

$\ddagger$ See Ref. 3 . for more details. 
${ }^{3} R$ is the scalar curvature in three dimensions. This is the time-time component of Eqs.(2). It represents a constraint at the classical level and the invariance under time reparametrization. Its quantum counterpart

$$
\mathcal{H} \Psi=0
$$

is the WDW equation. If we integrate over the hypersurface $\Sigma$ and we define

$$
\hat{\Lambda}_{\Sigma}=(2 \kappa) G_{i j k l} \pi^{i j} \pi^{k l}-\frac{\sqrt{g}}{2 \kappa}^{3} R
$$

Eq.(41) can be cast into the following form

$$
\int \mathcal{D}\left[g_{i j}\right] \Psi^{*}\left[g_{i j}\right]\left[\int_{\Sigma} d^{3} x \hat{\Lambda}_{\Sigma}\right] \Psi\left[g_{i j}\right]=-\frac{\Lambda_{c}}{\kappa} V \int \mathcal{D}\left[g_{i j}\right] \Psi^{*}\left[g_{i j}\right] \Psi\left[g_{i j}\right],
$$

where we have multiplied Eq.(44) by $\Psi^{*}\left[g_{i j}\right]$, we have functionally integrated over the three spatial metric $g_{i j}$ and where we have defined the volume of the hypersurface $\Sigma$ as $V=\int_{\Sigma} d^{3} x \sqrt{g}$. Thus one can formally re-write the WDW equation as

$$
\frac{1}{V} \frac{\int \mathcal{D}\left[g_{i j}\right] \Psi^{*}\left[g_{i j}\right] \int_{\Sigma} d^{3} x \hat{\Lambda}_{\Sigma} \Psi\left[g_{i j}\right]}{\int \mathcal{D}\left[g_{i j}\right] \Psi^{*}\left[g_{i j}\right] \Psi\left[g_{i j}\right]}=\frac{1}{V} \frac{\left\langle\Psi\left|\int_{\Sigma} d^{3} x \hat{\Lambda}_{\Sigma}\right| \Psi\right\rangle}{\langle\Psi \mid \Psi\rangle}=-\frac{\Lambda_{c}}{\kappa},
$$

We can gain more information considering a separation of the spatial part of the metric into a background term, $\bar{g}_{i j}$, and a quantum fluctuation, $h_{i j}$,

$$
g_{i j}=\bar{g}_{i j}+h_{i j}
$$

Thus Eq.(7) becomes

$$
\frac{1}{V} \frac{\left\langle\Psi\left|\int_{\Sigma} d^{3} x\left[\hat{\Lambda}_{\Sigma}^{(0)}+\hat{\Lambda}_{\Sigma}^{(1)}+\hat{\Lambda}_{\Sigma}^{(2)}+\ldots\right]\right| \Psi\right\rangle}{\langle\Psi \mid \Psi\rangle}=-\frac{\Lambda_{c}}{\kappa} \Psi\left[g_{i j}\right],
$$

where $\hat{\Lambda}_{\Sigma}^{(i)}$ represents the $i^{\text {th }}$ order of perturbation in $h_{i j}$. By observing that the kinetic part of $\hat{\Lambda}_{\Sigma}$ is quadratic in the momenta, we only need to expand the three-scalar curvature $\int d^{3} x \sqrt{g}^{3} R$ up to the quadratic order and we get

$$
\begin{aligned}
& \int_{\Sigma} d^{3} x \sqrt{\bar{g}}\left[-\frac{1}{4} h \triangle h+\frac{1}{4} h^{l i} \triangle h_{l i}-\frac{1}{2} h^{i j} \nabla_{l} \nabla_{i} h_{j}^{l}+\right. \\
& \left.+\frac{1}{2} h \nabla_{l} \nabla_{i} h^{l i}-\frac{1}{2} h^{i j} R_{i a} h_{j}^{a}+\frac{1}{2} h R_{i j} h^{i j}+\frac{1}{4} h\left(R^{(0)}\right) h\right]
\end{aligned}
$$

where $h$ is the trace of $h_{i j}$ and $R^{(0)}$ is the three dimensional scalar curvature onshell. Eq.(7) represents the Sturm-Liouville problem associated with the cosmological constant. The related boundary conditions are dictated by the choice of the trial wavefunctionals which, in our case are of the Gaussian type. Different types of wavefunctionals correspond to different boundary conditions. Extracting the TT tensor contribution from Eq.(7) approximated to second order in perturbation of the spatial part of the metric into a background term, $\bar{g}_{i j}$, and a perturbation, $h_{i j}$, we get

$$
\hat{\Lambda}_{\Sigma}^{\perp}=\frac{1}{4 V} \int_{\Sigma} d^{3} x \sqrt{\bar{g}} G^{i j k l}\left[(2 \kappa) K^{-1 \perp}(x, x)_{i j k l}+\frac{1}{(2 \kappa)}\left(\triangle_{2}\right)_{j}^{a} K^{\perp}(x, x)_{i a k l}\right] \text {. }
$$


Here $G^{i j k l}$ represents the inverse DeWitt metric and all indices run from one to three. The propagator $K^{\perp}(x, x)_{i a k l}$ can be represented as

$$
K^{\perp}(\vec{x}, \vec{y})_{i a k l}:=\sum_{\tau} \frac{h_{i a}^{(\tau) \perp}(\vec{x}) h_{k l}^{(\tau) \perp}(\vec{y})}{2 \lambda(\tau)},
$$

where $h_{i a}^{(\tau) \perp}(\vec{x})$ are the eigenfunctions of $\triangle_{2}$, whose explicit expression for the massive case will be shown in the next section. $\tau$ denotes a complete set of indices and $\lambda(\tau)$ are a set of variational parameters to be determined by the minimization of Eq.(11). The expectation value of $\hat{\Lambda}_{\Sigma}^{\perp}$ is easily obtained by inserting the form of the propagator into Eq.(11) and minimizing with respect to the variational function $\lambda(\tau)$. Thus the total one loop energy density for TT tensors becomes

$$
\frac{\Lambda}{8 \pi G}=-\frac{1}{4} \sum_{\tau}\left[\sqrt{\omega_{1}^{2}(\tau)}+\sqrt{\omega_{2}^{2}(\tau)}\right] .
$$

The above expression makes sense only for $\omega_{i}^{2}(\tau)>0$, where $\omega_{i}$ are the eigenvalues of $\triangle_{2}$.

\section{One loop energy Regularization and Renormalization for a $f(R)=R$ theory}

The Spin-two operator for the Schwarzschild metric in the Regge and Wheeler representation [4], leads to the following system of equations $(r \equiv r(x))$

$$
\left\{\begin{array}{l}
{\left[-\frac{d^{2}}{d x^{2}}+\frac{l(l+1)}{r^{2}}+m_{1}^{2}(r)\right] f_{1}(x)=\omega_{1, l}^{2} f_{1}(x)} \\
{\left[-\frac{d^{2}}{d x^{2}}+\frac{l(l+1)}{r^{2}}+m_{2}^{2}(r)\right] f_{2}(x)=\omega_{2, l}^{2} f_{2}(x)}
\end{array},\right.
$$

where reduced fields have been used and the proper geodesic distance from the throat of the bridge has been considered. Close to the throat, the effective masses are

$$
\left\{\begin{array}{c}
m_{1}^{2}(r) \simeq-m_{0}^{2}(M) \\
m_{2}^{2}(r) \simeq m_{0}^{2}(M)
\end{array}\right.
$$

where we have defined a parameter $r_{0}>2 M G$ and $m_{0}^{2}(M)=3 M G / r_{0}^{3}$. The main reason for introducing a new parameter resides in the fluctuation of the horizon that forbids any kind of approach. It is now possible to explicitly evaluate Eq.(13) in terms of the effective mass. To further proceed we use the W.K.B. method used by ' $t$ Hooft in the brick wall problem [5] and we count the number of modes with frequency less than $\omega_{i}, i=1,2$. Thus the one loop total energy for TT tensors becomes

$$
\frac{\Lambda}{8 \pi G}=\rho_{1}+\rho_{2}=-\frac{1}{16 \pi^{2}} \sum_{i=1}^{2} \int_{\sqrt{m_{i}^{2}(r)}}^{+\infty} \omega_{i}^{2} \sqrt{\omega_{i}^{2}-m_{i}^{2}(r)} d \omega_{i},
$$

where we have included an additional $4 \pi$ coming from the angular integration. Here, we use the zeta function regularization method to compute the energy densities $\rho_{1}$ and $\rho_{2}$. Note that this procedure is completely equivalent to the subtraction procedure 
of the Casimir energy computation where the zero point energy (ZPE) in different backgrounds with the same asymptotic properties is involved. To this purpose, we introduce the additional mass parameter $\mu$ in order to restore the correct dimension for the regularized quantities. Such an arbitrary mass scale emerges unavoidably in any regularization scheme. One gets

$$
\rho_{i}(\varepsilon)=\frac{m_{i}^{4}(r)}{256 \pi^{2}}\left[\frac{1}{\varepsilon}+\ln \left(\frac{\mu^{2}}{m_{i}^{2}(r)}\right)+2 \ln 2-\frac{1}{2}\right],
$$

$i=1,2$. The renormalization is performed via the absorption of the divergent part into the re-definition of the bare classical constant $\Lambda$

$$
\Lambda \rightarrow \Lambda_{0}+\Lambda^{\text {div }}
$$

The remaining finite value for the cosmological constant reads

$$
\begin{aligned}
& \frac{\Lambda_{0}}{8 \pi G}=\frac{1}{256 \pi^{2}}\left\{m_{1}^{4}(r)\left[\ln \left(\frac{\mu^{2}}{\left|m_{1}^{2}(r)\right|}\right)+2 \ln 2-\frac{1}{2}\right]\right. \\
& \left.+m_{2}^{4}(r)\left[\ln \left(\frac{\mu^{2}}{m_{2}^{2}(r)}\right)+2 \ln 2-\frac{1}{2}\right]\right\}=\left(\rho_{1}(\mu)+\rho_{2}(\mu)\right)=\rho_{\text {eff }}^{T T}(\mu, r) .
\end{aligned}
$$

The quantity in Eq.(19) depends on the arbitrary mass scale $\mu$. It is appropriate to use the renormalization group equation to eliminate such a dependence. To this aim, we impose that 6 ]

$$
\frac{1}{8 \pi G} \mu \frac{\partial \Lambda_{0}^{T T}(\mu)}{\partial \mu}=\mu \frac{d}{d \mu} \rho_{e f f}^{T T}(\mu, r) .
$$

Solving it we find that the renormalized constant $\Lambda_{0}$ should be treated as a running one in the sense that it varies provided that the scale $\mu$ is changing

$$
\Lambda_{0}(\mu, r)=\Lambda_{0}\left(\mu_{0}, r\right)+\frac{G}{16 \pi}\left(m_{1}^{4}(r)+m_{2}^{4}(r)\right) \ln \frac{\mu}{\mu_{0}} .
$$

Substituting Eq.(21) into Eq.(19) we find

$$
\frac{\Lambda_{0}\left(\mu_{0}, M\right)}{8 \pi G}=-\frac{1}{128 \pi^{2}}\left\{m_{0}^{4}(M)\left[\ln \left(\frac{m_{0}^{2}(M)}{4 \mu_{0}^{2}}\right)+\frac{1}{2}\right]\right\} .
$$

Eq.(22) has a maximum when $\$$

$$
\frac{1}{e}=\frac{m_{0}^{2}(M)}{4 \mu_{0}^{2}} \quad \Longrightarrow \quad \Lambda_{0}\left(\mu_{0}\right)=\frac{G m_{0}^{4}(M)}{32 \pi}=\frac{G \mu_{0}^{4}}{2 \pi e^{2}} .
$$

The computed cosmological constant appears to depend on the Schwarzschild radius. This dependence simply reflects the fact that the chosen background introduce one physical scale: the Schwarzschild radius. Nothing prevent us to consider a more general situation where the scalar curvature $R$ is replaced by a generic function of $R$. Therefore, we will consider the Sturm-Liouville problem of Eq.(17) in the context of a $f(R)$ theory $\llbracket$.

$\S$ Remark Note that in any case, the maximum of $\Lambda$ corresponds to the minimum of the energy density.

I| A recent review on the problem of $f(R)$ theories can be found in Ref. [7]. A more general discussion on modified gravities of the type $f(R), f(G)$ and $f(R, G)$ where $G$ is the Gauss-Bonnet invariant, can be found in Ref. 8 . 
3. One loop energy Regularization and Renormalization for a generic $f(R)$ theory in a Hamiltonian formulation

In this section, we report the main steps discussed in Ref. [9] for a $f(R)$ theory in connection with the Sturm-Liouville problem of Eq.(7). Although a $f(R)$ theory does not need a cosmological constant, rather it should explain it, we shall consider the following Lagrangian density describing a generic $f(R)$ theory of gravity

$$
\mathcal{L}=\sqrt{-g}(f(R)-2 \Lambda), \quad \text { with } f^{\prime \prime} \neq 0,
$$

where $f(R)$ is an arbitrary smooth function of the scalar curvature and primes denote differentiation with respect to the scalar curvature. A cosmological term is added also in this case for the sake of generality, because in any case, Eq.(24) represents the most general lagrangian to examine. Obviously $f^{\prime \prime}=0$ corresponds to GR. The generalized Hamiltonian density for the $f(R)$ theory assumes the form

$$
\begin{aligned}
& \mathcal{H}=\frac{1}{2 \kappa}\left[-\sqrt{g} f^{\prime}(R)\left({ }^{(3)} R-2 \Lambda_{c}-3 K_{i j} K^{i j}+K^{2}\right)\right. \\
& \left.+V(\mathcal{P})+2 g^{i j}\left(\sqrt{g} f^{\prime}(R)\right)_{\mid i j}-2 p^{i j} K_{i j}\right]
\end{aligned}
$$

where

$$
\mathcal{P}=-6 \sqrt{g} f^{\prime}(R)
$$

and

$$
V(\mathcal{P})=\sqrt{g}\left[R f^{\prime}(R)-f(R)\right] .
$$

Henceforth, the superscript 3 indicating the spatial part of the metric will be omitted on the metric itself. When $f(R)=R, V(\mathcal{P})=0$ as it should be. Eq.(25) becomes

$$
\begin{aligned}
& \mathcal{H}=f^{\prime}(R)\left[(2 \kappa) G_{i j k l} \pi^{i j} \pi^{k l}-\frac{\sqrt{g}}{2 \kappa}\left({ }^{(3)} R-2 \Lambda_{c}\right)\right] \\
& +\frac{1}{2 \kappa}\left[\sqrt{g} f^{\prime}(R)\left(2 K_{i j} K^{i j}\right)+V(\mathcal{P})+2 g^{i j}\left(\sqrt{g} f^{\prime}(R)\right)_{\mid i j}-2 p^{i j} K_{i j}\right] .
\end{aligned}
$$

Since

$$
p^{i j}=\sqrt{g} K^{i j}
$$

then we obtain

$$
\begin{aligned}
& \mathcal{H}=f^{\prime}(R)\left[(2 \kappa) G_{i j k l} \pi^{i j} \pi^{k l}-\frac{\sqrt{g}}{2 \kappa}\left({ }^{(3)} R-2 \Lambda_{c}\right)\right] \\
& +\frac{1}{2 \kappa}\left[2 \sqrt{g} K_{i j} K^{i j}\left(f^{\prime}(R)-1\right)+V(\mathcal{P})+2 g^{i j}\left(\sqrt{g} f^{\prime}(R)\right)_{\mid i j}\right]
\end{aligned}
$$

and transforming into canonical momenta, one gets

$$
\mathcal{H}=f^{\prime}(R)\left[(2 \kappa) G_{i j k l} \pi^{i j} \pi^{k l}-\frac{\sqrt{g}}{2 \kappa}\left({ }^{(3)} R-2 \Lambda_{c}\right)\right]
$$

I See Ref.[10] for technical details. 
The Cosmological Constant

$$
+2(2 \kappa)\left[G_{i j k l} \pi^{i j} \pi^{k l}+\frac{\pi^{2}}{4}\right]\left(f^{\prime}(R)-1\right)+\frac{1}{2 \kappa}\left[V(\mathcal{P})+2 g^{i j}\left(\sqrt{g} f^{\prime}(R)\right)_{\mid i j}\right] .
$$

By imposing the Hamiltonian constraint, we obtain

$$
\begin{aligned}
& f^{\prime}(R)\left[(2 \kappa) G_{i j k l} \pi^{i j} \pi^{k l}-\frac{\sqrt{g}}{2 \kappa}{ }^{(3)} R\right]+2(2 \kappa)\left[G_{i j k l} \pi^{i j} \pi^{k l}+\frac{\pi^{2}}{4}\right]\left(f^{\prime}(R)-1\right) \\
& +\frac{1}{2 \kappa}\left[V(\mathcal{P})+2 g^{i j}\left(\sqrt{g} f^{\prime}(R)\right)_{\mid i j}\right]=-f^{\prime}(R) \sqrt{g} \frac{\Lambda_{c}}{\kappa}
\end{aligned}
$$

If we assume that $f^{\prime}(R) \neq 0$ the previous expression becomes

$$
\begin{aligned}
& {\left[(2 \kappa) G_{i j k l} \pi^{i j} \pi^{k l}-\frac{\sqrt{g}}{2 \kappa}{ }^{(3)} R\right]+(2 \kappa)\left[G_{i j k l} \pi^{i j} \pi^{k l}+\frac{\pi^{2}}{4}\right] \frac{2\left(f^{\prime}(R)-1\right)}{f^{\prime}(R)}} \\
& +\frac{1}{2 \kappa f^{\prime}(R)}\left[V(\mathcal{P})+2 g^{i j}\left(\sqrt{g} f^{\prime}(R)\right)_{\mid i j}\right]=-\sqrt{g} \frac{\Lambda_{c}}{\kappa} .
\end{aligned}
$$

Now, we integrate over the hypersurface $\Sigma$ to obtain

$$
\begin{aligned}
& \int_{\Sigma} d^{3} x\left\{\left[(2 \kappa) G_{i j k l} \pi^{i j} \pi^{k l}-\frac{\sqrt{g}}{2 \kappa}{ }^{(3)} R\right]+(2 \kappa)\left[G_{i j k l} \pi^{i j} \pi^{k l}+\frac{\pi^{2}}{4}\right] \frac{2\left(f^{\prime}(R)-1\right)}{f^{\prime}(R)}\right\} \\
& +\int_{\Sigma} d^{3} x \frac{1}{2 \kappa f^{\prime}(R)}\left[V(\mathcal{P})+2 g^{i j}\left(\sqrt{g} f^{\prime}(R)\right)_{\mid i j}\right]=-\frac{\Lambda_{c}}{\kappa} \int_{\Sigma} d^{3} x \sqrt{g}
\end{aligned}
$$

The term

$$
\frac{1}{\kappa} \int_{\Sigma} d^{3} x \frac{1}{f^{\prime}(R)} g^{i j}\left(\sqrt{g} f^{\prime}(R)\right)_{\mid i j}
$$

appears to be a three-divergence and therefore will not contribute to the computation. The remaining equation simplifies into

$$
\begin{aligned}
& \int_{\Sigma} d^{3} x\left\{\left[(2 \kappa) G_{i j k l} \pi^{i j} \pi^{k l}-\frac{\sqrt{g}}{2 \kappa}{ }^{(3)} R\right]+(2 \kappa)\left[G_{i j k l} \pi^{i j} \pi^{k l}+\frac{\pi^{2}}{4}\right] \frac{2\left(f^{\prime}(R)-1\right)}{f^{\prime}(R)}\right. \\
& \left.+\frac{V(\mathcal{P})}{2 \kappa f^{\prime}(R)}\right\}=-\frac{\Lambda_{c}}{\kappa} \int_{\Sigma} d^{3} x \sqrt{g} .
\end{aligned}
$$

By a canonical procedure of quantization, we want to obtain the vacuum state of a generic $f(R)$ theory. By repeating the same procedure for the generalized WDW equation Eq.(37), we obtain

$$
\begin{aligned}
& \frac{1}{V} \frac{\left\langle\Psi\left|\int_{\Sigma} d^{3} x\left[\hat{\Lambda}_{\Sigma}^{(2)}\right]\right| \Psi\right\rangle}{\langle\Psi \mid \Psi\rangle}+\frac{2 \kappa}{V} \frac{2\left(f^{\prime}(R)-1\right)}{f^{\prime}(R)} \frac{\left\langle\Psi\left|\int_{\Sigma} d^{3} x\left[G_{i j k l} \pi^{i j} \pi^{k l}+\pi^{2} / 4\right]\right| \Psi\right\rangle}{\langle\Psi \mid \Psi\rangle} \\
& +\frac{1}{V} \frac{\left\langle\Psi\left|\int_{\Sigma} d^{3} x V(\mathcal{P}) /\left(2 \kappa f^{\prime}(R)\right)\right| \Psi\right\rangle}{\langle\Psi \mid \Psi\rangle}=-\frac{\Lambda_{c}}{\kappa}
\end{aligned}
$$

From Eq.(38), we can define a "modified" $\hat{\Lambda}_{\Sigma}^{(2)}$ operator which includes $f^{\prime}(R)$. Thus, we obtain

$$
\frac{\left\langle\Psi\left|\int_{\Sigma} d^{3} x\left[\hat{\Lambda}_{\Sigma, f(R)}^{(2)}\right]\right| \Psi\right\rangle}{\langle\Psi \mid \Psi\rangle}+\frac{\kappa}{V} \frac{\left(f^{\prime}(R)-1\right)}{f^{\prime}(R)} \frac{\left\langle\Psi\left|\int_{\Sigma} d^{3} x\left[\pi^{2}\right]\right| \Psi\right\rangle}{\langle\Psi \mid \Psi\rangle}
$$




$$
+\frac{1}{V} \frac{\left\langle\Psi\left|\int_{\Sigma} d^{3} x \frac{V(\mathcal{P})}{2 \kappa f^{\prime}(R)}\right| \Psi\right\rangle}{\langle\Psi \mid \Psi\rangle}=-\frac{\Lambda_{c}}{\kappa},
$$

where

$$
\hat{\Lambda}_{\Sigma, f(R)}^{(2)}=(2 \kappa) h(R) G_{i j k l} \pi^{i j} \pi^{k l}-\frac{\sqrt{g}}{2 \kappa}{ }^{3} R^{l i n}
$$

with

$$
h(R)=1+\frac{2\left[f^{\prime}(R)-1\right]}{f^{\prime}(R)}
$$

and where ${ }^{3} R^{\text {lin }}$ is the linearized scalar curvature whose expression is shown in square brackets of Eq.(10). Note that when $f(R)=R$, consistently it is $h(R)=1$. From Eq.(39), we redefine $\Lambda_{c}$

$$
\Lambda_{c}^{\prime}=\Lambda_{c}+\frac{1}{2 V} \frac{\left\langle\Psi\left|\int_{\Sigma} d^{3} x \frac{V(\mathcal{P})}{f^{\prime}(R)}\right| \Psi\right\rangle}{\langle\Psi \mid \Psi\rangle}=\Lambda_{c}+\frac{1}{2 V} \int_{\Sigma} d^{3} x \sqrt{g} \frac{R f^{\prime}(R)-f(R)}{f^{\prime}(R)}
$$

where we have explicitly used the definition of $V(\mathcal{P})$. In the same spirit of the previous section, we restrict the analysis to the contribution of physical degrees of freedom, namely TT tensors. $+*$. Thus Eq.(22) becomes

$$
\frac{\Lambda_{0}^{\prime}\left(\mu_{0}, r\right)}{8 \pi G}=-\frac{m_{0}^{4}(M)}{128 \pi^{2}}\left[\ln \left(\frac{m_{0}^{2}(M)}{4 \mu_{0}^{2}}\right)+\frac{1}{2}\right] .
$$

Now, we compute the maximum of $\Lambda_{0}^{\prime}$, by setting $x=m_{0}^{2}(M) / 4 \mu_{0}^{2}$. Thus $\Lambda_{0}^{\prime}$ becomes

$$
\Lambda_{0}^{\prime}\left(\mu_{0}, x\right)=-\frac{G \mu_{0}^{4}}{\pi} x^{2}\left[\ln (x)+\frac{1}{2}\right] .
$$

As a function of $x, \Lambda_{0}\left(\mu_{0}, x\right)$ vanishes for $x=0$ and $x=\exp \left(-\frac{1}{2}\right)$ and when $x \in\left[0, \exp \left(-\frac{1}{2}\right)\right], \Lambda_{0}^{\prime}\left(\mu_{0}, x\right) \geq 0$. It has a maximum for $\bar{x}=1 / e$ equivalent to $m_{0}^{2}(M)=4 \mu_{0}^{2} / e$ and its value is

$$
\Lambda_{0}^{\prime}\left(\mu_{0}, \bar{x}\right)=\frac{G \mu_{0}^{4}}{2 \pi e^{2}}
$$

or

$$
\frac{1}{\sqrt{h(R)}}\left[\Lambda_{0}\left(\mu_{0}, \bar{x}\right)+\frac{1}{2 V} \int_{\Sigma} d^{3} x \sqrt{g} \frac{R f^{\prime}(R)-f(R)}{f^{\prime}(R)}\right]=\frac{G \mu_{0}^{4}}{2 \pi e^{2}} .
$$

Isolating $\Lambda_{0}\left(\mu_{0}, \bar{x}\right)$, we get

$$
\Lambda_{0}\left(\mu_{0}, \bar{x}\right)=\sqrt{h(R)} \frac{G \mu_{0}^{4}}{2 \pi e^{2}}-\frac{1}{2 V} \int_{\Sigma} d^{3} x \sqrt{g} \frac{R f^{\prime}(R)-f(R)}{f^{\prime}(R)} .
$$

Note that $\Lambda_{0}\left(\mu_{0}, \bar{x}\right)$ can be set to zero when

$$
\sqrt{h(R)} \frac{G \mu_{0}^{4}}{2 \pi e^{2}}=\frac{1}{2 V} \int_{\Sigma} d^{3} x \sqrt{g} \frac{R f^{\prime}(R)-f(R)}{f^{\prime}(R)} .
$$

+ For a complete derivation of the effective action for a $f(R)$ theory, see Ref. 11].

* By a canonical decomposition of the gauge part $\xi_{a}$ into a transverse part $\xi_{a}^{T}$ with $\nabla^{a} \xi_{a}^{T}=0$ and a longitudinal part $\xi_{a}^{\|}$with $\xi_{a}^{\|}=\nabla_{a} \psi$, it is possible to show that most of the contribution comes from the longitudinal part (scalar). Evidence against scalar perturbation contribution in a Schwarzschild background has been discussed in Ref.[12]. 
Let us see what happens when $f(R)=\exp (-\alpha R)$. This choice is simply suggested by the regularity of the function at every scale. In this case, Eq.(48) becomes

$$
\sqrt{\frac{3 \alpha \exp (-\alpha R)+2}{\alpha \exp (-\alpha R)}} \frac{G \mu_{0}^{4}}{\pi e^{2}}=\frac{1}{\alpha V} \int_{\Sigma} d^{3} x \sqrt{g}(1+\alpha R) .
$$

For Schwarzschild, it is $R=0$, and by setting $\alpha=G$, we have the relation

$$
\mu_{0}^{4}=\frac{\pi e^{2}}{G} \sqrt{\frac{1}{(3 G+2) G}} .
$$

Like the case of $f(R)=R$, the purpose of this calculation is related to Eq.(7), which can be applied to different backgrounds, i.e. de Sitter, Schwarzschild-de Sitter, etc. The result of this process will be a spectrum built in terms of vacuum fluctuations. Once the "Ground State" of this spectrum will be identified, then there will be a chance to have an approach for explaining the so-called Dark Energy. Note that in this approach, there is no evolution in time, so some pathologies of the type "Big Rip" do not come into play at this stage.

\section{References}

[1] For a pioneering review on this problem see S. Weinberg, Rev. Mod. Phys. 61, 1 (1989). For more recent and detailed reviews see V. Sahni and A. Starobinsky, Int. J. Mod. Phys. D 9, 373 (2000), astro-ph/9904398 N. Straumann, The history of the cosmological constant problem gr-qc/0208027; T.Padmanabhan, Phys.Rept. 380, 235 (2003), hep-th/0212290.

[2] B. S. DeWitt, Phys. Rev. 160, 1113 (1967).

[3] R.Garattini, J. Phys. A 39, 6393 (2006); gr-qc/0510061. R. Garattini, J.Phys.Conf.Ser. 33, 215 (2006); gr-qc/0510062.

[4] T. Regge and J. A. Wheeler, Phys. Rev. 108, 1063 (1957).

[5] G. 't Hooft, Nucl. Phys. B 256, 727 (1985).

[6] J.Perez-Mercader and S.D. Odintsov, Int. J. Mod. Phys. D 1, 401 (1992). I.O. Cherednikov, Acta Physica Slovaca, 52, (2002), 221. I.O. Cherednikov, Acta Phys. Polon. B 35, 1607 (2004). M. Bordag, U. Mohideen and V.M. Mostepanenko, Phys. Rep. 353, 1 (2001). Inclusion of nonperturbative effects, namely beyond one-loop, in de Sitter Quantum Gravity have been discussed in S. Falkenberg and S. D. Odintsov, Int. J. Mod. Phys. A 13, 607 (1998); hep-th 9612019.

[7] S. Capozziello and M. Francaviglia, Extended Theories of Gravity and their Cosmological and Astrophysical Applications, arXiv:0706.1146,

[8] S. Nojiri and S. D. Odintsov, Int.J.Geom.Meth.Mod.Phys. 4, 115 (2007); hep-th/0601213.

[9] S. Capozziello and R. Garattini, Class.Quant.Grav. 24, 1627 (2007); gr-qc/0702075.

[10] L. Querella, Variational Principles and Cosmological Models in Higher-Order Gravity - Ph.D. Thesis. gr-qc/9902044.

[11] G. Cognola, E. Elizalde, S. Nojiri, S. D. Odintsov and S. Zerbini, JCAP 050210 (2005); hep-th/0501096.

[12] R. Garattini, TSPU Vestnik 44 N7, 72 (2004); gr-qc/0409016.

[13] M.C.B. Abdalla, S. Nojiri and S. D. Odintsov, Class.Quant.Grav. 22, L35 (2005); hep-th/0409177. 